\title{
纳米多孔金膜表面等离子体共振效应的理论分析和传感应用
}

\author{
王 丽 ${ }^{1,2}$ 逯丹凤 ${ }^{1}$ 高 然 $^{1}$ 程 进 ${ }^{1}$ 张 喆 $^{3}$ 祁志美 ${ }^{1, *}$ \\ ( ${ }^{1}$ 中国科学院电子学研究所, 传感技术国家重点实验室, 北京 $100190 ;{ }^{2}$ 中国科学院大学, 北京 100049 ; \\ ${ }^{3}$ 北京交通大学计算机与信息技术学院, 北京 100044)
}

\begin{abstract}
摘要: 纳米多孔金膜(NPGF)化学和热力学稳定性好, 比表面积大, 具有显著的表面等离子体共振 (SPR) 效应, 适宜于用作SPR生化传感芯片。本文对NPGF的SPR效应进行了理论分析, 得到了在NPGF/空气 界面传播的表面等离子体色散曲线, 获得了优化NPGF-SPR传感性能所需的最佳薄膜厚度约为 $60 \mathrm{~nm}$; 在此基础上利用溅射沉积一化学脱合金两步法在玻璃基板上制备出大面积均匀的超薄NPGF, 采用 Krestchmann棱镜稞合结构测试了NPGF在可见-近红外波段的SPR共振光谱及其传感特性, 通过利用菲 涅耳公式并结合Bruggeman介电常数近似理论对测得的共振波长进行拟合, 得出NPGF的孔隙率约为 0.38。未经修饰的NPGF是亲水薄膜, 能够有效富集水中的双酚A, 使得NPGF-SPR传感器对双酚A的探 测下限达到 $5 \mathrm{nmol} \cdot \mathrm{L}^{-1}$; 经过疏水化处理后, NPGF对非极性苯并萠分子的富集能力获得显著增强, 使得 传感器对苯并萠的探测下限达到 $1 \mathrm{nmol} \cdot \mathrm{L}^{-1}$ 。
\end{abstract}

关键词：纳米多孔金膜；传播表面等离子体共振；富集；高灵敏度；苯并芘

中图分类号: 0647

\section{Theoretical Analyses and Chemical Sensing Application of Surface Plasmon Resonance Effect of Nanoporous Gold Films}

\author{
WANG Li ${ }^{1,2}$ LU Dan-Feng ${ }^{1} \quad$ GAO Ran ${ }^{1}$ CHENG Jin ${ }^{1}$ \\ ZHANG Zhe ${ }^{3} \quad$ QI Zhi-Mei ${ }^{1, *}$
}

( ${ }^{1}$ State Key Laboratory of Transducer Technology, Institute of Electronics, Chinese Academy of Sciences, Beijing 100190, P. R. China; ${ }^{2}$ University of Chinese Academy of Sciences, Beijing 100049, P. R. China; ${ }^{3}$ School of Computer and Information Technology, Beijing Jiaotong University, Beijing 100044, P. R. China)

\begin{abstract}
Nanoporous gold films (NPGFs) are chemically robust and thermally stable, have large specific area and salient surface plasmon resonance (SPR) effect. Due to these features, NPGFs are quite applicable for high-sensitivity SPR sensors. In this study, the SPR effect of NPGFs was theoretically analyzed and the dispersion relation was obtained for propagating surface plasmons at the NPGF/air interface. The optimal thickness of NPGF required for optimizing its SPR sensing performance was determined to be $\sim 60 \mathrm{~nm}$. Large-area, uniform and ultrathin NPGFs were prepared by a two-step approach involving sputtering deposition and chemical dealloying. The SPR resonance band in the visible-near-infrared region and the sensing properties of NPGFs were measured with the Kretschmann prism-coupling configuration. Porosity of the NPGF was determined to be $\sim 0.38$ by fitting the measured
\end{abstract}

Received: December 15, 2016; Revised: February 27, 2017; Published online: February 28, 2017.

*Corresponding author. Email: zhimei-qi@mail.ie.ac.cn; Tel: +86-10-58887196.

The project was supported by the National Key Basic Research Program of China (973) (2015CB352100), National Natural Science Foundation of China (61377064, 61401432, 61401019, 61675203), and Research Equipment Development Project of Chinese Academy of Sciences (YZ201508).

国家重点基础研究发展规划项目(973) (2015CB352100), 国家自然科学基金(61377064, 61401432，61401019，61675203)和中国科学院科研装备研制项 目(YZ201508)资助

(C) Editorial office of Acta Physico-Chimica Sinica 
resonance wavelengths based on a combination of the Fresnel formula and the Bruggeman dielectric constant approximation theory. Since the non-modified NPGFs are hydrophilic and enable effective enrichment of bisphenol A (BPA) in water, the NPGF-SPR sensor can easily detect BPA at concentrations as low as $5 \mathrm{nmol} \cdot \mathrm{L}^{-1}$. After hydrophobilization of NPGFs, the sensor enables detection of trace amounts of benzo[a]pyrene $(\mathrm{BaP})$ in water, with the detection limit being $1 \mathrm{nmol} \cdot \mathrm{L}^{-1}$.

Key Words: Nanoporous gold film; Propagating surface plasmon resonance; Enrichment; High sensitivity; Benzo[a]pyrene

\section{1 引 言}

纳米多孔金膜(NPGF)是一种先进功能材料, 拥有热力学高度稳定的三维纳米孔-孔互通结构 以及独特的物理和化学特性, 在催化、化工、新 能源、生化传感器、表面增强拉曼光谱技术(SERS) 等领域具有广阔的应用前景, 已经引起国内外学 者的广泛关注 ${ }^{1-7}$ 。在可见-近红外波段, NPGF的 孔尺寸远小于波长, 因此是光学均匀薄膜, 具有 显著的表面等离子体共振(SPR)效应, 能够用于制 备SPR生化传感器。值得指出的是这里所说的表面 等离子体是指能够在NPGF表面传播的表面等离 激元, 不是与其纳米孔结构相关的局域表面等离 子体。基于NPGF的SPR传感器容许薄膜内部的消 逝场与薄膜内部富集的待测分子相互作用, 从而 将作用深度从常规SPR传感器的表面单分子层厚 度扩展至整个NPGF厚度, 这种作用深度的扩展意 味着传感器灵敏度的提升和探测下限的降低。尽 管拥有高灵敏度等优点, 基于NPGF的SPR传感器 却鲜有报道，原因之一是受其制备方法的制约。 NPGF的传统制备方法是将市售的金银合金箔片 浸入浓硝酸中通过化学反应除去合金中的银原 子, 使得金原子重新排列组装形成三维无序多孔 结构。这种方法称为脱合金法 ${ }^{8-13}$, 为了方便使用, 所制得的自立式NPGF需要从溶液中转移到玻璃 或硅基片上。为了增强NPGF与基片的附着力, 还 需要对基片表面进行化学修饰。这种传统的NPGF 制备方法不能调控薄膜厚度, 不适合在基片表面 制备大面积均匀的NPGF, 也不容易获得数十纳米 厚的超薄NPGF。因此, 传统的箔片脱合金法不利 于制备NPG薄膜器件, 尤其不适合那些需要精确 控制NPGF厚度的薄膜器件。SPR传感器是一种典 型的薄膜器件, 其性能与功能薄膜的厚度密切相 关。对于致密金膜SPR传感器, 金膜厚度控制在 50 $\mathrm{nm}$ 才能获得良好的传感特性。本文的仿真结果指 出当NPGF用于SPR传感器时, 理想的薄膜厚度约 为 $60 \mathrm{~nm}$ 。利用传统的箔片脱合金法目前还不能实 现这种超薄NPGF。为了克服传统制备方法的缺 点, 研究组在前期工作中提出了射频溅射沉积-化
学脱合金两步法, 成功制备出大面积均匀的超薄 NPGF, 采用数纳米厚的铬膜作为过渡层, 有效增 强了NPGF与玻璃基底的附着力, 但是在脱合金过 程中需要格外小心地控制腐蚀时间, 以防铬膜被 腐蚀而引起薄膜脱落。研究组最近对制备方法进 行了改进，采用了铬/金双层金属膜作为过渡层有 效抑制了在脱合金过程中NPGF的脱落现象, 而且 容许通过延长脱合金时间对NPGF的孔尺寸进行 调控。

本文对NPGF的SPR效应进行了理论分析, 为 实验优化NPGF的SPR传感性能提供了依据; 利用 改进的两步法制备出超薄NPGF, 测试了NPGF在 可见-近红外波段的SPR共振光谱, 通过仿真拟合 实验数据, 获得了NPGF的孔隙率, 进一步实验分 析了NPGF-SPR传感器对水中双酚 $\mathrm{A}(\mathrm{BPA})$ 和苯并 萠(BaP)的响应特性。

\section{2 实验部分}

\section{1 试剂与仪器}

苯并萠/甲醇标准样品溶液 (benzo[a]pyrene/ methanol, $5.26 \mu \mathrm{g} \cdot \mathrm{mL}^{-1}$, HPLC)购自中国计量科学 院, 用于配制待测样品溶液; 硝酸(纯度 $68 \%(w$, 质量分数) 购自国药集团有限公司, 用于腐蚀芯片 以制备纳米多孔金薄膜; 双酚 $\mathrm{A}$ 和正十二硫醇(纯 度 $98 \%$ )购自美国Aladdin公司; 无水乙醇(纯度 $\geq$ 99.7\%)购自北京化工厂, 用于配制正十二硫醇/乙 醇溶液; 实验中使用的去离子水经过Milli-Q纯净 水机二次净化。金银合金靶购自北京有色金属技 术开发中心有限公司, 靶材中金和银的质量比为 $1: 1$.

厚度为 $1 \mathrm{~mm}$ 的玻璃祄底购自日本Matsunami 株式会社; 角度为 $45^{\circ} / 45^{\circ} / 90^{\circ}$ 的玻璃棱镜购自北京 北东光电自动化开发公司(折射率 $n_{\mathrm{p}}=1.799 @ \lambda=$ $633 \mathrm{~nm})$; HR 4000型电荷耦合器件 $(\mathrm{CCD})$ 光谱分析 仪购自美国Ocean Optics公司; 多模石英光纤购自 浙江雷畴科技有限公司; 透镜和线性偏振片购自 北京大恒光电技术公司; 用于制作样品测试槽的 硅橡胶薄板购自昆山新富阳塑胶材料有限公司; 
蠕动百购自保定兰格恒流洜有限公司。

\section{2 芯片制备}

采用射频浌射沉积-化学脱合金两步法在玻 璃基板上制备了大面积均匀的NPGF。首先, 利用 射频溅射技术在清洗干净的玻璃基板上依次溅射 约 $3 \mathrm{~nm}$ 厚的铬膜、约 $10 \mathrm{~nm}$ 厚的金膜和约 $60 \mathrm{~nm}$ 厚 的金银合金膜; 其次, 在室温下将制得的多层金 属膜芯片浸入浓硝酸中进行脱合金处理, $4 \mathrm{~min}$ 后 将芯片快速取出, 并用足量的去离子水反复冲洗 以除去残留的硝酸, 然后用氮气吹干。经硝酸处 理后薄膜的颜色由最初的银白色变为棕色, 意味 着NPGF的形成。NPGF的颜色来自于其局域等离 子体共振效应。前面已经指出, 铬膜的作用是增 加玻璃祄底和上层金属之间的结合力, 金膜的作 用是在脱合金过程中保护铬膜，防止其被硝酸腐 蚀。最后得到的NPGF芯片非常稳定, 容许在水溶 液中长时间浸泡和反复使用。

\section{3 实验装置}

图1显示了本文使用的基于Kretschmann棱镜 耦合结构的波长检测型NPGF-SPR传感装置。 NPGF芯片的玻璃基底一侧通过折射率匹配的耦 合液紧贴在棱镜底面, 由硅橡胶薄板制作的样品 槽通过挤压与NPGF密封接触。卤铇灯发出的宽带 入射光依次经过光纤、聚焦透镜和线性偏振片后 变为 $p$ 偏振平行光束 (发散角小于 $0.2^{\circ}$ )。该平行光 束射入玻璃棱镜并在玻璃基底与金属膜的界面发 生全反射, 全反射产生的消逝场穿透NPGF并与 $\mathrm{NPGF} /$ 空气界面上的表面等离子体相互作用, 在 特定波长下两者发生共振耦合, 产生能量转移, 使得反射光谱中在相应的共振波长处呈现一波 谷。通过利用 $\mathrm{CCD}$ 光谱仪记录反射光谱, 就能实 验测得共振波长。在实验过程中, 通过调节图1所 示的 $\theta$ 角实现了对共振波长初始值的选择。

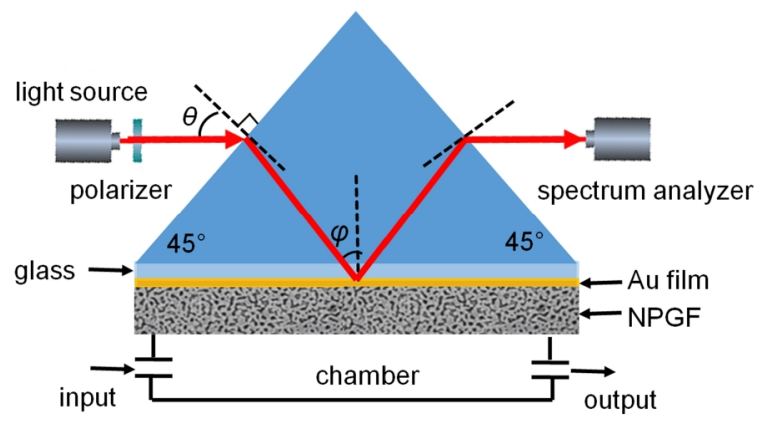

图1 波长检测型NPGF-SPR传感器测试平台示意图

Fig.1 Schematic diagram of the wavelengthinterrogated NPGF-SPR sensor platform NPGF: nanoporous gold film

\section{4 理论分析}

NPGF在可见-近红外波段是光学均匀薄膜, 其平均复折射率 $\left(n=n^{\prime}+\mathrm{i} n^{\prime \prime}\right)$ 能够借助Bruggeman 介电常数近似方程求解得出 ${ }^{14}$ :

$$
f_{1} \frac{n_{1}^{2}-n^{2}}{n_{1}{ }^{2}+2 n^{2}}+f_{2} \frac{n_{2}^{2}-n^{2}}{n_{2}{ }^{2}+2 n^{2}}+f_{3} \frac{n_{3}^{2}-n^{2}}{n_{3}{ }^{2}+2 n^{2}}=0
$$

式中 $f_{1} 、 f_{2} 、 f_{3}$ 依次为NPGF中金骨架、孔及孔内吸 附物的体积分数, 且 $f_{1}+f_{2}+f_{3}=1 ; n_{1} 、 n_{2} 、 n_{3}$ 分 别为以上三种物质的折射率; $f_{2}+f_{3}$ 即为 NPGF的 孔隙率。为了简化分析, 基于方程(1)计算了不同 孔隙率的NPGF在空气中 $\left(n_{2}=1\right)$ 且不含吸附物 $\left(f_{3}=\right.$ 0 )时的复折射率。图2(a)和2(b)分别显示了NPGF复 折射率的实部 $\left(n^{\prime}\right)$ 和虚部 $\left(n^{\prime \prime}\right)$ 在可见-近红外波段 的色散曲线, 图中每一条曲线对应一个给定的孔 隙率。孔隙率为 0 的曲线是文献给出的纯金的复折 射率色散曲线。从图2(b)可以看出随着孔隙率的增 大, NPGF复折射率的虚部逐渐减小, 意味着其吸 光能力逐渐减弱。利用图 2 中的NPGF复折射率数 据, 基于公式(2)所示的传播表面等离子体色散方 程 $^{15}$, 可以求得在 NPGF/空气界面传播的表面等离 子体的色散曲线。

$$
\beta_{\mathrm{SP}}=\frac{\omega}{c} \sqrt{\frac{\varepsilon_{a} \varepsilon_{m}}{\varepsilon_{a}+\varepsilon_{m}}}
$$
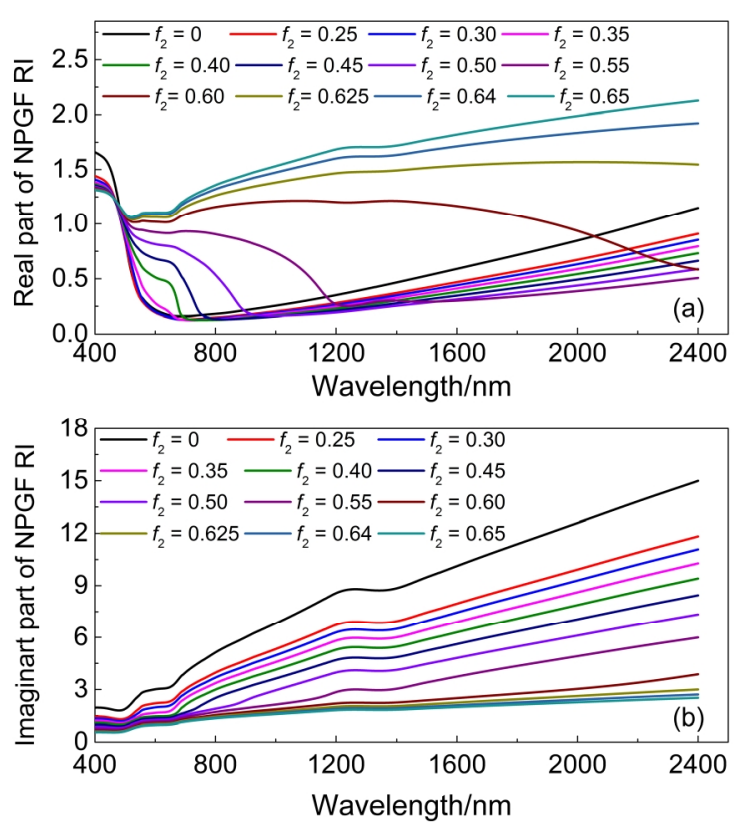

图 2 不同孔隙率的NPGF复折射率随波长的变化

Fig.2 Complex refractive index (RI) versus wavelength for NPGFs with different porosities (a) real part; (b) imaginary part 
式中 $\beta_{\mathrm{SP}}$ 为表面等离子体波的传播常数, $\omega$ 为光的 角频率, $c$ 为真空中的光速, $\varepsilon_{\mathrm{a}}=1$ (空气的介电常 数), $\varepsilon_{\mathrm{m}}$ 为 NPGF的介电常数的实部 $\left(\varepsilon_{\mathrm{m}}=n^{\prime 2}-n^{\prime \prime 2}\right)$ 。 图3显示了 9 条表面等离子体色散曲线 $\left(\mathrm{a}_{1}\right.$ 至 $\left.\mathrm{a}_{9}\right)$, 对 应于 9 个孔隙率。从图中可知随着 NPGF孔隙率的 增大, 表面等离子体波的色散曲线逐渐下移。图3 还给出了光在空气中和在棱镜中的色散曲线 $\left(\mathrm{b}_{1}\right.$ 和 $\mathrm{b}_{3}$ )(对于空气, $\beta=\omega / c$; 对于棱镜, $\beta=n_{\mathrm{p}} \omega / c$ )。利 用Kretschmann棱镜耦合结构在NPGF/空气界面激 发的表面等离子体波, 其传播常数 $\beta_{\mathrm{SP}}$ 位于两曲线 $b_{1}$ 和 $b_{3}$ 之间。因此从图3中可以看出随着NPGF孔隙 率的增大, $\beta_{\mathrm{SP}}$ 的取值范围越来越窄; 当孔隙率超 过0.55时, 理论上只能在近红外波段激发表面等离 子体波。按照相位匹配条件, 只有当入射光在棱 镜底面的波矢分量等于表面等离子体波的传播常 数时才能发生二者的共振耦合。为了在色散空间 中理解相位匹配条件, 图3给出了 $\beta_{x}=(\omega / c) n_{\mathrm{p}} \sin \varphi$ 在入射角 $\varphi=45^{\circ}$ 时对应的曲线 $\mathrm{b}_{2}$ 。该曲线与 $\mathrm{a}_{1}$ 至 $\mathrm{a}_{9}$ 中的任一曲线都相交, 在每一个交点处的 $(\beta, \omega)$ 满 足位相匹配条件, 这意味着在孔隙率不超过 0.64 的情况下, 采用Kretschmann结构在入射角为 $\varphi=$ $45^{\circ}$ 时能够在NPGF/空气界面共振激励传播表面等 离子体, 但是发生共振的色散范围随着孔隙率的 增大而逐渐变窄。上述理论分析有助于从孔隙率 的角度实现NPGF-SPR性能的优化。

利用Fresnel多层膜反射公式仿真分析了不含 吸附物的NPGF在空气中的SPR 共振光谱(即 $f_{3}=$ $\left.0, n_{2}=1\right)$ 。图4(a)显示了在给定 $f_{2}=0.3$ 和入射角 $\theta=$ $-14^{\circ}$ 的条件下计算得出的反射光谱。从图中可以 看出每一个反射谱包含一个形状规则的波谷, 谷

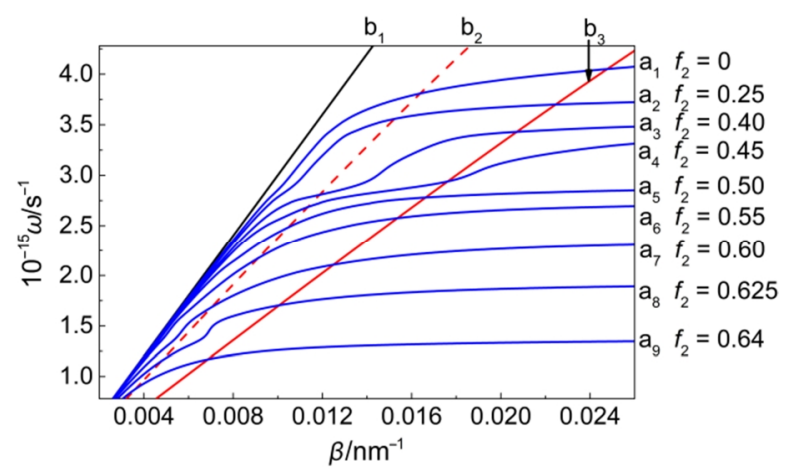

图3NPGF/空气界面的表面等离子体色散曲线 $\left(a_{1}-a_{9}\right)$, 光在空气 $\left(b_{1}\right)$ 和在棱镜 $\left(b_{3}\right)$ 中的色散曲线以及全内反射 (TIR)产生的消逝波的色散曲线 $\left(\mathrm{b}_{2}, \lambda\right.$ 射角 $\left.\varphi=45^{\circ}\right)$

Fig.3 Dispersions for surface plasmons at the NPGF/air interface $\left(a_{1}-a_{9}\right)$ and light in air $\left(b_{1}\right)$ and in prism $\left(b_{3}\right)$ and evanescent wave produced at $\varphi=45^{\circ}$ with $\operatorname{TIR}\left(b_{2}\right)$
底位置(即共振波长)几乎不随NPGF厚度的变化而 移动, 只是波谷的深度和宽度随着厚度的变化而 变化。厚度为 $60 \mathrm{~nm}$ 的NPGF对应的波谷既深又窄, 有利于准确确定共振波长及其变化量。另一方面, 以本文实验中的被测对象 $\mathrm{BaP}\left(n_{3}=1.887\right)^{16}$ 为例, 设定在NPGF内吸附的 $\mathrm{BaP}$ 分子所占的体积分数为 $f_{3}=0.1$, 仿真分析了 $\mathrm{BaP}$ 吸附导致的 $\mathrm{SPR}$ 共振波长 变化量 $\Delta \lambda_{\mathrm{R}}$ [这里 $\Delta \lambda_{\mathrm{R}}=\lambda_{\mathrm{R}}\left(f_{3}=0.1\right)-\lambda_{\mathrm{R}}\left(f_{3}=0\right)$ ]。图 $4(b)$ 给出了在不同孔隙率下 $\Delta \lambda_{\mathrm{R}}$ 随NPGF厚度的变 化曲线。从图中可看出当孔隙率不超过 0.4 时, 对 于任意孔隙率的NPGF, 在 40-60 nm的厚度区间内 $\Delta \lambda_{\mathrm{R}}$ 随着厚度的增大逐渐升高, 当厚度超过 $60 \mathrm{~nm}$ 时, $\Delta \lambda_{R}$ 趋于稳定。综合图4(a)和4(b)的仿真结果可 看出 $60 \mathrm{~nm}$ 厚的NPGF不仅有利于准确确定共振波 长及其变化量, 而且能够导致理想的灵敏度。按 照前期研究结果 ${ }^{17}$, 对于一个 $60 \mathrm{~nm}$ 厚的NPGF, $f_{3}=$ 0.1 意味着, 如果将吸附的所有 $\mathrm{BaP}$ 分子组成一个 与NPGF等面积的致密薄膜, 该薄膜的厚度可达到 $6 \mathrm{~nm}$, 远大于 $\mathrm{BaP}$ 单分子层厚度。以上理论分析结
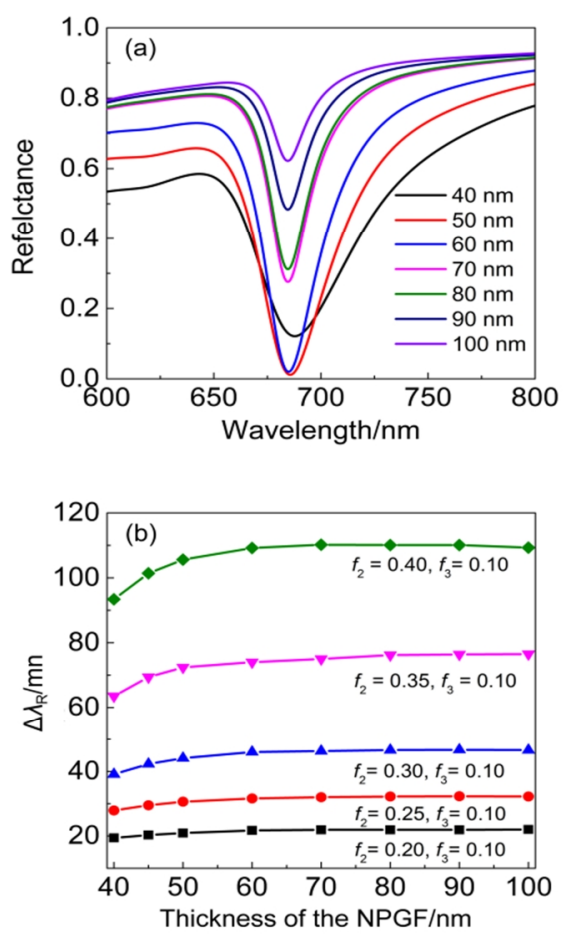

图 4 (a)在 $f_{2}=0.3$ 与 $f_{3}=0$ 的条件下仿真得到的不同厚度 NPGF的菲涅耳反射光谱; (b) 在 $f_{3}=0.1$ 时仿真得出的不同 孔隙率NPGF的共振波长变化量与膜厚的关系

Fig.4 (a) Fresnel reflectance spectra for NPGFs of different thicknesses simulated with $f_{2}=0.3$ and $f_{3}=0$;

(b) Thickness dependence of resonant wavelength shift

for NPGFs of different porosities with $f_{3}=0.1$ 
果为从NPGF厚度方面优化设计NPGF-SPR传感器 提供了指导。

\section{4 结果与讨论}

\section{1 纳米多孔金薄膜的表征}

通过扫描电子显微镜(SEM)观察NPGF表面形 貌, 结果如图5所示。由图可知, 薄膜呈现分布均 匀的三维无序孔状结构。这种结构十分有利于小 分子的快速扩散和吸附。

利用自建的波长检测型NPGF-SPR传感器, 测 试了在不同入射角下NPGF传感芯片在空气中的 传播等离子体共振光谱。结果表明当NPGF暴露于 空气中时, 它在可见-近红外波段具有尖锐的传播 表面等离子体共振峰, 如图6(a)所示, 说明实验制 得的NPGF具有良好的光学质量和较高的品质因 子。为了获取NPGF的孔隙率, 利用Fresnel公式结 合Bruggeman介电常数近似理论对实验测得的共 振光谱进行了最佳拟合, 图6(b)显示了计算得到的 共振光谱, 三个角度对应的共振波长与各自的实 验值近似相同, 由此得出 NPGF的孔隙率近似为 0.38 。

\subsection{BPA小分子的吸附特性研究}

本文采用离位方法测试了NPGF对小分子的 吸附响应特性。首先研究了未经修饰的NPGF对水 溶液中BPA分子的吸附特性。采用去离子水配制 浓度为 5-150 $\mathrm{nmol} \cdot \mathrm{L}^{-1}$ 的一系列 BPA待测样品溶 液, 并记录入射角 $\theta=-12$ 时 NPGF在空气中的反 射光谱, 初始共振波长为704.98 nm。然后通过蠕 动泵将 $10 \mathrm{~mL}$ 浓度为 $5 \mathrm{nmol} \cdot \mathrm{L}^{-1}$ 的 $\mathrm{BPA}$ 待测样品溶 液通入样品槽中, 静置吸附达到平衡后, 将样品 溶液永出, 并对NPGF进行干燥处理, 充分去除被 吸附的水分子。再次记录NPGF的反射光谱, 共振 波长红移至 $705.87 \mathrm{~nm}$, 共振波长变化量 $\left(\Delta \lambda_{\mathrm{R}}\right)$ 为

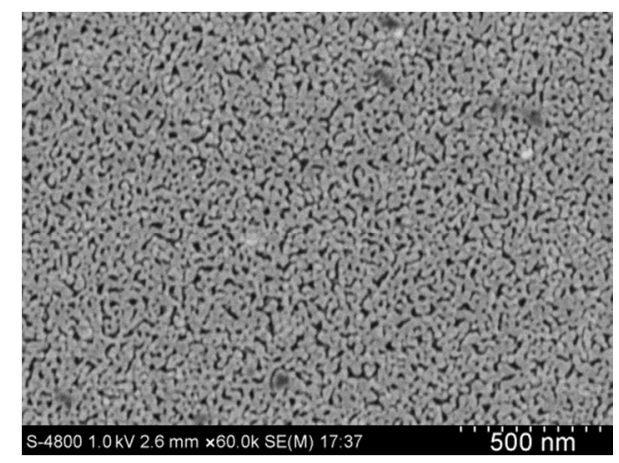

图5 NPGF扫描电子显微镜 (SEM)照片

Fig.5 Scanning electron microscope (SEM) image of the NPGF
$0.89 \mathrm{~nm}$, 本文使用的光谱仪刚好能分辨此光谱移 动, 表明该NPGF-SPR传感器对BPA的探测下限约 为 $5 \mathrm{nmol} \cdot \mathrm{L}^{-1}$ 。随后以同样的方法将 $\mathrm{BPA}$ 待测样品 溶液按照浓度从低到高的顺序依次通入样品槽中 进行测试。

图7(a)显示共振波长随着BPA水溶液浓度的 增加而不断红移。图7(b)为 $\Delta \lambda_{\mathrm{R}}$ 与 $\mathrm{BPA}$ 水溶液浓度 之间的关系曲线。由图可知, $\Delta \lambda_{\mathrm{R}}$ 随着浓度的增加 逐渐增大, 当 $\mathrm{BPA}$ 水溶液的浓度为 $150 \mathrm{nmol} \cdot \mathrm{L}^{-1}$ 时, $\Delta \lambda_{\mathrm{R}}$ 为 $17.74 \mathrm{~nm}$ 。这一高灵敏度一方面是因为 NPGF的大比表面积提供了更多的分子结合位点, 使得BPA分子可以在多孔层内大量富集; 另一方 面是因为表面电磁波与 BPA分子的作用深度从单 分子层扩展至了整个NPGF的厚度。

$\mathrm{BaP}$ 为非极性小分子, 与金膜表面的作用非常 弱, 因此不易吸附到金膜表面上, 为了促进 $\mathrm{BaP}$ 分子的吸附, 需要对NPGF内外表面进行疏水化修 饰 ${ }^{18-20}$ 。金膜表面的修饰方法非常成熟, 主要基于 硫-金化学键作用, 修饰常用的颈基化合物包括烷 基硫醇、颈基乙酸、和颈基取代环糊精等 ${ }^{21,22}$ 。本 文采用正十二烷基硫醇通过一步浸泡法修饰芯片 表面。首先记录NPGF修饰之前在入射角 $\theta=-14^{\circ}$ 时的共振光谱, 之后在室温下将芯片置于 40 $\mathrm{mmol} \cdot \mathrm{L}^{-1}$ 的正十二硫醇/乙醇溶液中浸泡修饰 24 $\mathrm{h}$, 使正十二硫醇分子中的放基 (一SH) 与NPGF中 的 $\mathrm{Au}$ 充分反应。然后取出芯片, 并用足量乙醇反 复冲洗以充分去除薄膜表面残留的硫醇分子并用 氮气吹干。最后测试NPGF修饰后的共振光谱, 如 图8所示。由图可知, NPGF经疏水化处理后, 其 共振波长发生了红移, 变化量约 $20 \mathrm{~nm}$, 结果表明

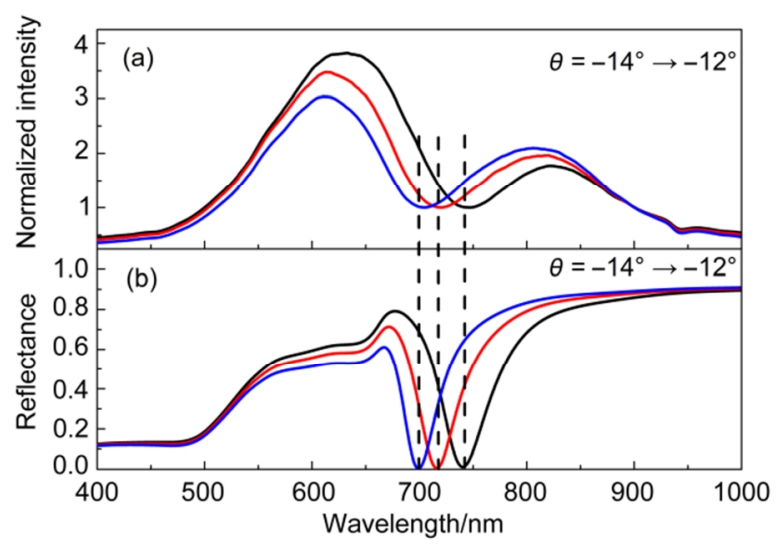

图6 (a)实验测得的NPGF在空气中的SPR光谱; (b)利用Fresnel公式计算得到的相应的共振光谱

Fig.6 (a) Measured and (b) simulated SPR spectra of the NPGF 

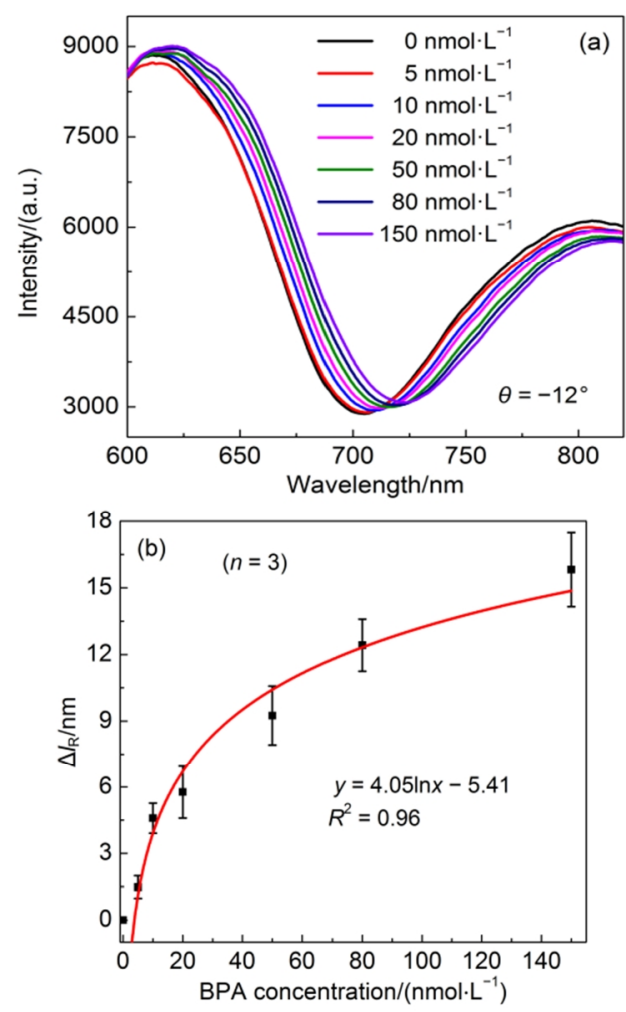

图 7 (a) 在不同BPA浓度下测得的 NPGF的SPR光谱;

(b) 共振波长变化量 $\left(\Delta \lambda_{R}\right)$ 与 $\mathrm{BPA}$ 溶液浓度的关系

Fig.7 (a) SPR spectra of the NPGF measured at different BPA concentrations; (b) resonant wavelength shifts $\left(\Delta \lambda_{R}\right)$ as a function of the BPA concentration

正十二硫醇中的巯基 $(-\mathrm{SH})$ 与 $\mathrm{NPGF}$ 中的 $\mathrm{Au}$ 形成 了稳定的共价键。

在正十二硫醇修饰 NPGF后, 本文研究了 NPGF-SPR芯片对不同浓度 BaP水溶液的吸附特 性。首先用去离子水稀释苯并萠/甲醇标准样品溶 液, 以配制浓度为 $1-50 \mathrm{nmol} \cdot \mathrm{L}^{-1}$ 的BaP水溶液, 并 记录NPGF未吸附BaP分子时 $\left(0 \mathrm{nmol} \cdot \mathrm{L}^{-1}\right)$ 在空气中

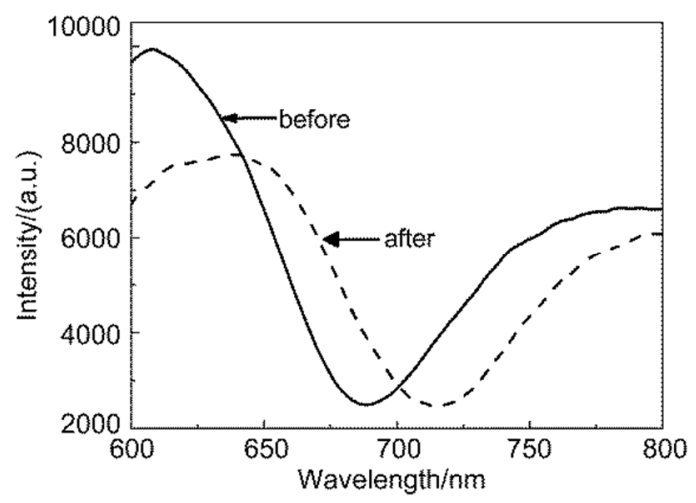

图8 正十二硫醇修饰前后的NPGF在空气中的SPR光谱

Fig.8 SPR spectra for the NPGF in air measured before and after modification with 1-dodecanethiol
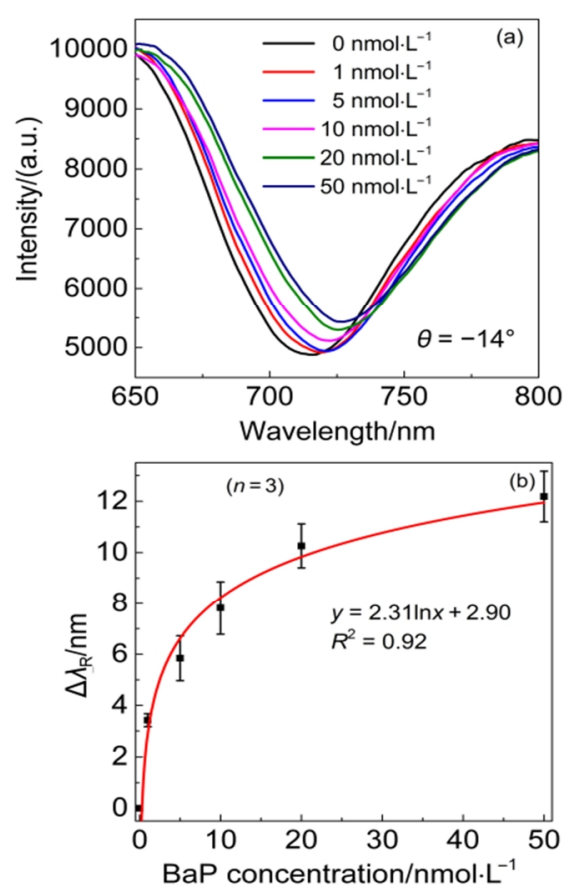

图9 (a) 在不同BaP浓度下测得的 NPGF的SPR光谱;

(b) 共振波长变化量与 $\mathrm{BaP}$ 溶液浓度的关系

Fig.9 (a) SPR spectra of the dodecanethiolmodified NPGF measured at different BaP concentrations; (b) Resonant wavelength shifts as a function of the BaP concentration

的共振光谱, 共振波长为 $716.07 \mathrm{~nm}$ 。之后使用蠕 动葲将 $10 \mathrm{~mL}$ 浓度为 $1 \mathrm{nmol} \cdot \mathrm{L}^{-1}$ 的 $\mathrm{BaP}$ 水溶液葲入 样品槽中, 待 $\mathrm{BaP}$ 吸附达到平衡后, 将 $\mathrm{BaP}$ 水溶液 泵出, 并对NPGF进行充分干燥。最后记录NPGF 在空气中的共振光谱, 此时共振波长红移至719.62 $\mathrm{nm}$ 。随后以同样的方法将 $\mathrm{BaP}$ 水溶液按照浓度从 低到高的顺序依次通入样品槽中进行吸附测试。 其中, 在 $\mathrm{BaP}$ 水溶液进出样品槽及浸泡吸附测试过 程中, 光源保持关闭状态以避免强光入射导致部 分 $\mathrm{BaP}$ 分子发生分解。图9(a) 为浸泡吸附不同浓度 的 $\mathrm{BaP}$ 水溶液后 NPGF在空气中的 SPR光谱。由图 可知, 随着 $\mathrm{BaP}$ 水溶液浓度的增加, 共振波长发生 红移。图9(b)给出了 $\Delta \lambda_{\mathrm{R}}$ 随 $\mathrm{BaP}$ 水溶液浓度的变化 趋势及拟合曲线 $\left(R^{2}=0.92\right)$ 。由图可知, $\Delta \lambda_{\mathrm{R}}$ 与 $\mathrm{BaP}$ 水溶液浓度成正相关, 并且当 $\mathrm{BaP}$ 水溶液浓度为 50 $\mathrm{nmol} \cdot \mathrm{L}^{-1}$ 时, $\Delta \lambda_{\mathrm{R}}$ 为 $11.07 \mathrm{~nm}$ 。这表明NPGF-SPR传 感器对水溶液中的 $\mathrm{BaP}$ 分子具有良好的响应。

\section{5 结 论}

本文对NPGF的SPR传感特性进行了系统的理 论和实验研究。在理论方面, 获得了不同孔隙率 的NPGF的表面等离子体波的色散曲线, 总结出优 
化NPGF-SPR生化传感器所需的最佳膜厚约为 60 $\mathrm{nm}$ 。在实验方面, 通过射频溅射与脱合金两步法 相结合制备了NPGF-SPR传感芯片, 利用自建的波 长检测型SPR传感装置检测了NPGF在空气中的反 射光谱, 基于Fresnel公式和Bruggeman介电常数近 似理论对实验测得的数据进行最佳拟合, 确定了 NPGF的孔隙率约为 0.38 , 并研究了NPGF-SPR传 感器对水溶液中的 $\mathrm{BPA}$ 和BaP两种小分子的吸附 响应特性。实验结果表明, NPGF未经修饰时, 传 感器对水溶液中的 BPA 分子的探测下限为 5 $\mathrm{nmol} \cdot \mathrm{L}^{-1}$; 当对 $\mathrm{NPGF}$ 的表面进行疏水化修饰后, 传感器对水溶液中的 $\mathrm{BaP}$ 分子实现了 $1 \mathrm{nmol} \cdot \mathrm{L}^{-1}$ 的 高灵敏检测。这种传感器的高灵敏度源于以下两 个方面: (1) 纳米多孔金薄膜的三维无序孔状结构 提供了大量的分子结合位点, 使得多孔层内可富 集更多目标分子; (2) NPGF内部消逝场与目标分 子的作用深度从单分子吸附层扩展至了整个 NPGF层厚度。另外, 由于NPGF表面容易通过化 学修饰方法固定颈基 $(-\mathrm{SH})$ 、氨基 $\left(-\mathrm{NH}_{2}\right)$ 等官能 团, 可进一步提高传感器的灵敏度。本文研制的 NPGF-SPR传感器对水体环境中的痕量有机污染 物探测具有重要意义。

\section{References}

(1) Sun, Q.; Ueno, K.; Yu, H.; Kubo, A.; Matsuo, Y.; Misawa, H. Light Sci. Appl. 2013, 2, e118. doi: 10.1038/1sa.2013.74

(2) Ruffato, G.; Romanato, F.; Garoli, D.; Cattarin, S. Opt. Exp. 2011, 19 (14), 13164. doi: 10.1364/OE.19.013164

(3) Fan, M.; Wang, Z. Y.; Liu, C. J. Acta Phys. -Chim. Sin. 2017, 33 (2), 435. [方 敏, 王宗元, 刘昌俊. 物理化学学报, 2017, 33 (2), 435.] doi: 10.3866/PKU.WHXB201611101

(4) Njoki, P. N.; Lim, I. S.; Mott, D.; Park, H. Y.; Khan, B.; Mishra, S.; Sujakumar, R.; Luo, J.; Zhong, C. J. Phys. Chem. C 2007, 111 (40),14664. doi: 10.1021/jp074902z

(5) Zhang, Z.; Lu, D. F.; Qi, Z. M. Acta Phys. -Chim. Sin. 2013, 29 (4), 868. [张 喆, 逯丹凤, 祁志美. 物理化学学报, 2013, 29 (4), 868.] doi: 10.3866/PKU.WHXB201302222

(6) Wang, Z.; Yang, M.; Chen, C.; Zhang, L.; Zeng, H. Sci. Report 2016,
6, 29611. doi: 10.1038/srep29611.

(7) Biener, J.; Nyce, G. W.; Hodge, A. M.; Biener, M. M.; Hamza, A. V.; Maier, S. A. Adv. Mater. 2008, 9999, 1. doi: 10.1002/adma.200701899.

(8) Detsi, E.; Punzhin, S.; Rao, J.; Onck, P. R.; De Hosson, J. T. M. ACS Nano 2012, 6, 3734. doi: $10.1021 / \mathrm{nn} 300179 \mathrm{n}$

(9) Feng, J.; Wu, J. Small 2012, 8, 3786. doi: 10.1002/small.201201591

(10) Fang, C.; Bandaru, N. M.; Ellis, A. V.; Voelcker, N. H. J. Mater. Chem. 2012, 22, 2952. doi: 10.1039/c2jm14889g

(11) Cherevko, S.; Chung, C. H. Electrochem. Commun. 2011, 13, 16. doi: 10.1016/j.elecom.2010.11.001

(12) Qian, L. H.; Chen, M. W. Appl. Phys. Lett. 2007, 91 (8), 083105. doi: http//dx.doi.org/10.1063/1.2773757

(13) Fujita, T.; Guan, P.; Mckenna, K.; Lang, X.; Hirata, A.; Zhang, L.; Tokunaga, T.; Arai, S.; Yamamoto, Y.; Tanaka, N.; Ishikawa,Y.; Asao, N.; Yamamoto, Y.; Erlebacher, J.; Chen, M. Nat. Mater. 2012, 11 (9), 775. doi: 10.1038/nmat3391

(14) Qi, Z. M.; Honma, I.; Zhou, H. Anal. Chem. 2006, 78, 1034. doi: $10.1021 / \mathrm{ac} 051380 \mathrm{f}$.

(15) Raether, H. Surface Plasmons on Smooth and Rough Surfaces and Gratings; Springer: Berlin Heidelberg, 1988, pp 4-13.

(16) http//www.chemspider.com/Chemical-Structure.2246.html.

(17) Wang, X.; Hao, W; Zhang, H.; Pan, Y.; Kang, Y.; Zhang, X.; Zou, M.; Tong, P.; Du, Y. Spectrochim. Acta Part A, Mol. Biomol. Spectrosc. 2015, 139, 215. doi: 10.1016/j.saa.2014.11.104

(18) Zhang, Z.; Lu, D. F.; Qi, Z. M. J. Phys. Chem. C 2012, 116, 3342. doi: $10.1021 / \mathrm{jp} 2102429$.

(19) Bao, L.; Sheng, P.; Li, J.; Wu, S.; Cai, Q.; Yao, S. Analyst 2012, 137, 4010. doi: 10.1039/C2AN35589B

(20) Fu, S.; Guo, X.; Wang, H.; Yang, T.; Wen, Y. Sens. Actuators B 2015, 212, 200. doi: 10.1016/j.snb.2015.01.134

(21) Feng, A.; Duan, J. M.; Du, J. J.; Jing, C. Y. Environ. Chem. 2014, 1 (33), 47. [冯 艾, 段晋明, 杜晶晶, 景传勇. 环境化学, 2014, 1 (33), 47.] doi: 10.7524/j.issn.0254-6108.2014.01.001

(22) Xie, Y. F.; Wang, X.; Ruan, W. D.; Song, W.; Zhao, B. Spectrosc. Spect. Anal. 2011, 31 (9), 2320. [谢云飞, 王 旭， 阮伟东, 宋 薇, 赵 冰. 光谱学与光谱分析, 2011, 31 (9), 2320.] 